\section{CALEFACCIÓN SOLAR EN EDIFICACIONES CON ACUMULACIÓN EN GRAN RESERVORIO DE AGUA}

\author{
Luis E. Juanicó ${ }^{1}$ y Alejandro D. González ${ }^{2}$
}

\section{Resumen}

Este trabajo muestra la factibilidad de calefacción solar en Bariloche (3.600 grados-día/año, $8.4^{\circ} \mathrm{C}$ media anual) empleando colectores tipo heat pipe y almacenamiento en un reservorio de agua aislado y utilizarlo mediante piso radiante. Mediante modelos térmicos dinámicos, se optimiza el sistema para una demanda anual de $13.000 \mathrm{kWh}$, que representa tanto una típica vivienda unifamiliar económica de $50 \mathrm{~m}^{2}$ (sin aislación térmica, $\left.260 \mathrm{kWh} / \mathrm{m}^{2}\right)$ u otra de $100 \mathrm{~m}^{2}$ con aislamiento $\left(130 \mathrm{kWh} / \mathrm{m}^{2}\right)$. Esta demanda se podría satisfacer con cinco colectores estándar de 20 tubos (2.088 $\mathrm{m}^{2}$ de área apertura) y un gran tanque de $63 \mathrm{~m}^{3} \mathrm{a}$ un costo de USD 27.000; pero también instalando ocho colectores se reduciría el tanque a $11 \mathrm{~m}^{3}$ y la

\section{SOLAR HEATING IN BUILDINGS BY USING A LARGE STORAGE WATER TANK}

Luis E. Juanicó ${ }^{1}$ and Alejandro D. González²

\section{Abstract}

This paper shows the feasibility of implementing solar heating in Bariloche (3,600 degrees/day, $8.4^{\circ} \mathrm{C}$ mean annual temperature) through heat pipe collectors and water contained in insulated water tanks for further use through floor heating systems. Dynamic thermal models are used to optimize the system in order to meet $13,000 \mathrm{kWh}$ annual demand, which represents both a $50 \mathrm{~m} 2$ single-family, non-insulated affordable housing unit $(260 \mathrm{kWh} / \mathrm{m} 2)$ or a $100 \mathrm{~m} 2$ insulated unit $(130$ $\mathrm{kWh} / \mathrm{m} 2$.) Such a demand could be met through five standard collectors, each containing 20 pipes (2,088m2, opening area), and a $63 \mathrm{~m} 3$ tank at a cost of USD 27,000 or eight collectors and an $11 \mathrm{~m} 3$ tank at a cost of USD 11,650. This paper discusses the 
inversión a 11.650 USD. Se discuten los motivos por los cuales, en Bariloche, siendo su temperatura media similar a la de países desarrollados de zona fría, se obtienen soluciones mucho más económicas: a) uso de colectores de vacío en lugar de planos; b) recurso solar y demanda de calefacción más distribuidos en el tiempo; c) tipo de reservorio escogido facilita su adecuada aislación y bajo coste. Se concluye que existe potencial para la aplicación de esta tecnología en amplia región de Sudamérica con demanda de calefacción.

\section{PALABRAS CLAVE: CALEFACCIÓN SOLAR, ALMACENAMIENTO DE CALOR, SUSTENTABILIDAD ENERGÉTICA DE VIVIENDAS.}

Recibido: 16-01-2017

Aceptado: 05-06-2018

1 Argentina. Instituto Andino Patagónico en Tecnologías Biológicas y Geoambientales (IPATEC), CONICET y Universidad Nacional del Comahue. ORCID: https://orcid.org/0000-0003-2422647X. Correo electrónico: juanico@comahue-conicet.gob.ar.

2 Argentina. Instituto Andino Patagónico en Tecnologías Biológicas y Geoambientales (IPATEC), CONICET y Universidad Nacional del Comahue. ORCID: https://orcid.org/0000-0002-02340960. Correo electrónico: gonzalezad@comahue-conicet.gob.ar reasons why Bariloche, whose mean temperature is similar to that of developed countries located in cold areas, achieves more economical solutions such as: a) the use of vacuum collectors instead of flat-plate collectors; b) properly distributed solar resources and heat demand; and c) an affordable type of water tank that enables proper insulation. This study concludes there is potential for the implementation of this technology in large parts of South America requiring heating solutions.

\section{KEYWORDS: SOLAR HEATING, HEAT STORAGE, ENERGY SUSTAINABILITY FOR HOUSING.}

Received: 16-01-2017

Accepted: 05-06-2018

1 Argentina. Andean-Patagonian Institute of Biological and GeoEnvironmental Technologies (IPATEC), CONICET and Comahue National University. ORCID: https://orcid.org/0000-0003-2422647X.Email: juanico@comahue-conicet.gob.ar.

2 Argentina. Andean-Patagonian Institute of Biological and GeoEnvironmental Technologies (IPATEC), CONICET and Comahue National University. ORCID: https://orcid.org/0000-0002-02340960. Email: gonzalezad@comahue-conicet.gob.ar

ARTíCULO: Solar heating in buildings by using a large storage water tank/Luis E. Juanicó y Alejandro D. González 


\section{Introducción}

En hogares de la ciudad patagónica de Bariloche (42 `S) el consumo más importante de energía (80\%) corresponde a calefacción; dado su clima frío y alta latitud, su resolución por medio de colectores solares implica un gran desafío. Sin embargo, el estudio de casos en Canadá y Alemania muestra que es factible, aunque costoso ( $\mathrm{Rad} \&$ Fung, 2016; Bär et al., 2015; Bauer et al., 2010). En estos antecedentes se capta el recurso solar con colectores planos conectados a un enorme reservorio que almacena calor en verano y lo extrae en invierno.

En Canadá, desde 2007 el proyecto Okotoks (Sibbit et al., 2012) provee el 95\% de la calefacción a 52 casas $\left(7.000 \mathrm{~m}^{2}, 135 \mathrm{~m}^{2} \mathrm{c} / \mathrm{u}\right)$ muy bien aisladas (117 kWh/m²/año con clima muy frío de temperatura media $4.4{ }^{\circ} \mathrm{C}$ y 5.020 grados-día de calefacción). Se utilizan 800 colectores solares planos $\left(2.312 \mathrm{~m}^{2}\right)$ y acumulación de largo plazo calentando el subsuelo mediante 144 pozos de 40 $\mathrm{m}$ de profundidad desplegados en $700 \mathrm{~m}^{2}$, y con acumulación de corto plazo mediante dos tanques aislados $\left(240 \mathrm{~m}^{3}\right)$ donde el agua alcanza $80^{\circ} \mathrm{C}$, siendo luego distribuida para proveer calefacción por piso radiante Cada año, la radiación solar incidente sobre los colectores inclinados $45^{\circ}$ (13.902 GJ) es parcialmente captada (4.391 GJ, eficiencia 31.6\%) y transferida al reservorio por un intercambiador (eficiencia 98.6\%) y por otro al sistema de calefacción. La demanda de calefacción se encuentra muy concentrada (55\%) en invierno y su recurso solar también; siendo su promedio anual similar a Bariloche $\left(3,8 \mathrm{kWh} / \mathrm{m}^{2} /\right.$ día $)$, su aporte en otoño-invierno es más bajo $(<1,7 \mathrm{kWh} /$ $\mathrm{m}^{2}$ por 4 meses $\mathrm{y}<0,9 \mathrm{kWh} / \mathrm{m}^{2}$ por 2 meses). Siendo más benigno el clima en Sudamérica, es esperable que aquí pudiera aplicarse esta tecnología, sin embargo, por su elevada inversión (USD 173.000 por vivienda) la misma ha sido descartada.

El proyecto alemán de Friedrichshaften (Bauer et al., 2010) desde 1996 utiliza un enorme tanque de agua enterrado $\left(12.000 \mathrm{~m}^{3}\right)$, construido con losa de $60 \mathrm{~cm}$ de concreto, para satisfacer el 25\% de la calefacción de un edificio multifamiliar (23.000 $\mathrm{m}^{2}, 100 \mathrm{kWh} / \mathrm{m}^{2} / \mathrm{año}$ ) mediante $4.050 \mathrm{~m}^{2}$ de colectores planos. La estrategia de maximizar el tanque para reducir su relación área/volumen reconoce, sin embargo, pérdidas del $40 \%$ debido a que la gran profundidad (20 metros) y peso del tanque impiden aislar su tercio inferior. Sumado a pérdidas del 8\% en la distribución, plantea dudas sobre la conveniencia de un gran sistema comunitario. Por último, se observaron bajas eficiencias (30\%) en los colectores, y se reconoce que esta es muy sensible a la temperatura, siendo casi nulo el rendimiento invernal (Rad \& Fung, 2016).

Por otra parte, desde 2006 el proyecto irlandés de Galway (Colclough \& Griffiths, 2016) utiliza un tanque de agua pequeño $\left(23 \mathrm{~m}^{3}\right)$ enterrado y muy 
bien aislado (60 cm EPS) junto con 6 de colectores de 20 tubos de vacío c/u (500 €) para calefaccionar una vivienda unifamiliar pasiva (1.350 kWh/año). La inversión en colectores (3.900 €) incluye 900€ de instalación, lo cual es una motivación (a instalar más colectores) del proyecto. La moderada inversión (12 mil euros) se evalúa competitiva frente a la opción eléctrica 5 .

En este trabajo se propone calefaccionar una vivienda en Bariloche por piso radiante, mediante colectores solares heat pipe (alta eficiencia y bajo coste) y gran tanque de agua. Esperamos que las mejores condiciones locales (demanda de calefacción y recurso solar más distribuidos) permitan obtener inversiones asequibles.

\section{Materiales y métodos}

\section{CONDICIONES CLIMÁTICAS}

En Bariloche la irradiación solar anual promedio es $3,8 \mathrm{kWh} / \mathrm{m}^{2} /$ día y con mínimas promedio invernal de 1,5 $\mathrm{kWh} / \mathrm{m}^{2} /$ día (figura la) (Grossi y Righini, 2007). La temperatura media anual es de $8,4^{\circ} \mathrm{C}$ y con mínimas media mensual $>2{ }^{\circ} \mathrm{C}$ (figura 1, izquierda. Servicio Meteorológico Nacional, 2017). Esto conduce a 3.600 grados-día por año de calefacción.
Comparando con otras locaciones donde se implementa esta tecnología, por ejemplo Okotoks, se observa que la irradiación solar anual es similar a Bariloche, pero en esta se encuentra más distribuida. La fracción invernal de demanda de calefacción es menor en Bariloche (38\%) en comparación a Okotoks (Sibbit et al., 2012) (55\%), donde la temperatura media invernal es menor a $-5^{\circ} \mathrm{C}$.

\section{DEMANDA EN CALEFACCIÓN}

Estudios previos muestran que el $97 \%$ de las viviendas en Bariloche carecen de aislamientos térmicos adecuados (González, Carlsson-Kanyama, Crivelli \& Gortari, 2007; González, 2014); como en toda la Patagonia (Díaz y Czjakowski, 2006), esto se debe en parte a históricos subsidios al precio del gas (González, 2009 y 2011), que incentivaron la resolución de la calefacción mediante mayor consumo. En Bariloche, el consumo anual de la casa unifamiliar promedio $\left(100 \mathrm{~m}^{2}\right)$ es de $5.000 \mathrm{~m}^{3}$ de gas natural, utilizando el $80 \%$ en calefacción. En el $85 \%$ de las viviendas se utilizan calefactores de tiro balanceado; siendo estos de eficiencia media (62\%) (Juanicó \& González, 2008a y 2008b) conducen a una necesidad de gas natural en calefacción de $2.600 \mathrm{~m}^{3} /$ año, equivalente a $26.000 \mathrm{kWh} /$ año (260 kWh/m²/año) de energía neta. En cambio, rehabilitando esta vivienda con una aislación 

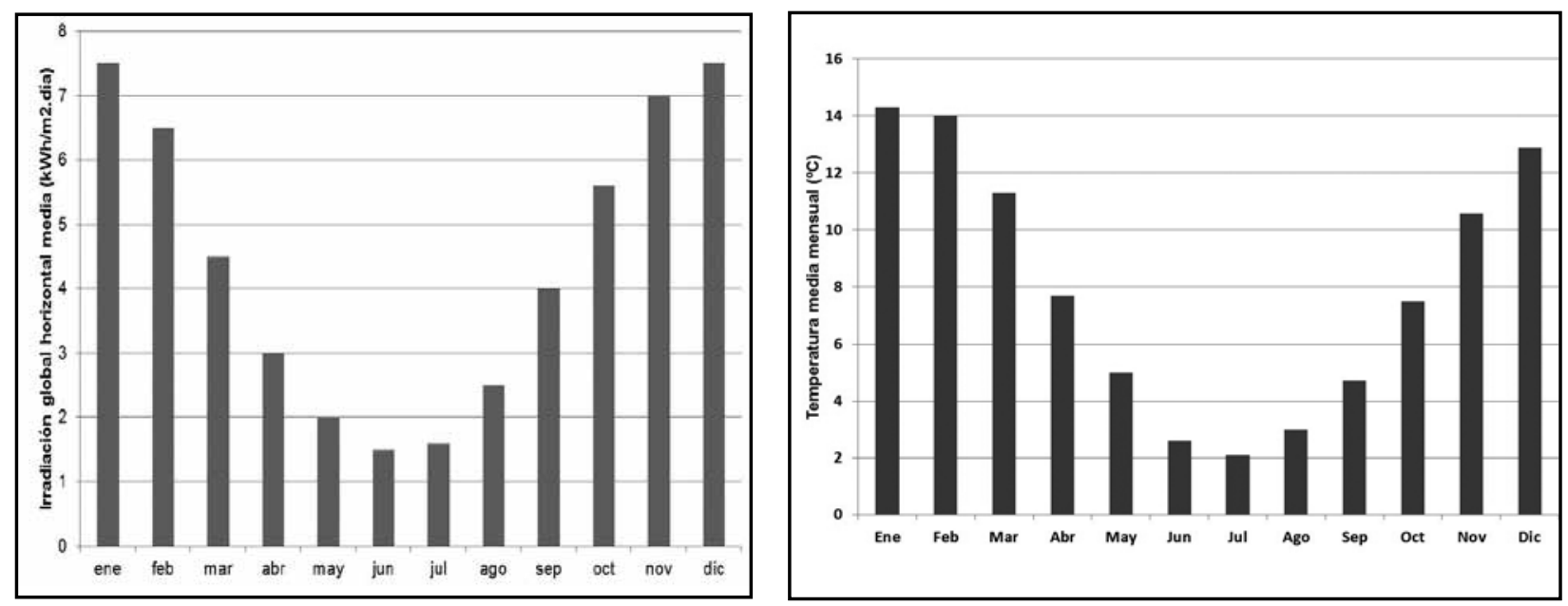

Figura 1. Izquierda: Irradiación global horizontal media, en $\mathrm{kWh} / \mathrm{m}^{2} /$ día en Bariloche. Derecha: Temperatura media mensual en Bariloche.

térmica moderada ( $6 \mathrm{~cm}$ en paredes y $12 \mathrm{~cm}$ en techos) se reduciría el consumo a la mitad (130 $\mathrm{kWh} / \mathrm{m}^{2} / \mathrm{año}$ ) con una inversión aproximada de 8 mil dólares (400 $\mathrm{m}^{2}$ de placas EPS de costo 5 USD/ $\mathrm{m}^{2}$, revestimiento cementicio de $5 \mathrm{USD} / \mathrm{m}^{2}$, soportes y mano de obra). Como será discutido, esta rehabilitación es conveniente y, en adelante, asumi-

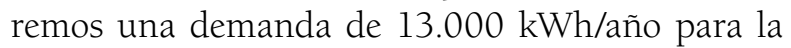
casa promedio $\left(100 \mathrm{~m}^{2}\right)$; sin embargo, esta también representaría una vivienda económica típica de 50 $\mathrm{m}^{2}$ sin rehabilitación, $\mathrm{u}$ otra de $200 \mathrm{~m}^{2}$ con diseño bioclimático (65 kWh/m²/año). Es decir, se diseña aquí el sistema para una demanda de 13.000 kWh/ año y el tamaño de la vivienda que encuentre satisfecha su necesidad de calefacción dependerá de su calidad térmica.

En base a los grados-día de calefacción mensual (base $18.3^{\circ} \mathrm{C}$ ) calculados con las temperaturas medias ilustradas en la figura 1 (derecha), se obtienen las fracciones mensuales de demanda de calefacción (tabla 1). 
TABLA 1. FRACCIÓN MENSUAL DE DEMANDA DE CALEFACCIÓN PARA BARILOCHE.

\begin{tabular}{lr}
\hline & \% del consumo anual \\
\hline Enero & 3,3 \\
\hline Febrero & 3,2 \\
\hline Marzo & 5,6 \\
\hline Abril & 8,0 \\
\hline Mayo & 10,8 \\
\hline Junio & 12,1 \\
\hline Julio & 13,1 \\
Agosto & 12,6 \\
\hline Septiembre & 10,7 \\
\hline Octubre & 8,7 \\
\hline Noviembre & 6,7 \\
\hline Diciembre & 5,2 \\
\hline Anual & $\mathbf{1 0 0} \%$
\end{tabular}

Fuente:Autores.

\section{SISTEMA PROPUESTO}

Proponemos el uso de colectores solares tipo heat pipe para alimentar un sistema de calefacción por piso radiante, mediante un reservorio de agua aislado y enterrado. Esta solución permite utilizar tanques de bajo costo (rotomoldeados de fibra de vidrio), reducir el uso del terreno e impacto visual.
En suelos blandos (típicos de la zona) la excavación se realiza a bajo costo. Por otra parte, si bien es cierto que un tanque mayor presentaría una superficie menor relativa al volumen, podemos obtener muy buena aislación $\left(U=0.1 \mathrm{~W} / \mathrm{m}^{2} /{ }^{\circ} \mathrm{C}\right)$ con $35 \mathrm{~cm}$ de EPS, un aislante económico pero aplicable bajo tanques de hasta 12 metros de altura (en suelos densidad $1,6 \mathrm{~g} / \mathrm{cm}^{3}$, siendo la presión máxima 2 $\mathrm{kg} / \mathrm{cm}^{2}$ ).

Calentando hasta $90{ }^{\circ} \mathrm{C}$ la mezcla agua-glicol en colectores y empleando un sistema de calefacción de baja temperatura $\left(28^{\circ} \mathrm{C}\right)$, maximizamos la energía térmica útil del reservorio para una temperatura máxima de $85{ }^{\circ} \mathrm{C}$ y mínima de $33{ }^{\circ} \mathrm{C}$, considerando saltos de $5{ }^{\circ} \mathrm{C}$ en ambos intercambiadores de calor. Un estudio detallado de los intercambiadores está fuera del alcance de este trabajo, pero comentaremos que este salto térmico permite transferir las potencias requeridas $(\sim 10 \mathrm{~kW})$ con intercambiadores comerciales tubulares de cobre de bajo costo ("BOWA Solution", 2017), 200 USD c/u, y razonables caudales de recirculación. Por ejemplo, para un único colector se recomienda un caudal de 500 litros/hora, el cual permite transferir $3 \mathrm{~kW}$ con el salto térmico propuesto. Finalmente, es posible reducir la estratificación del reservorio $\left(<2^{\circ} \mathrm{C}\right)$ ubicando el intercambiador solar por debajo del de calefacción (Colclough \& Griffiths, 2016). 


\section{MODELO TÉRMICO-SOLAR DE COLECTORES SOLARES}

La eficiencia de un colector solar se aproxima linealmente por su eficiencia óptica o máxima, $\eta_{0}$, y el término lineal de pérdidas de calor, calculado según su coeficiente de pérdidas, $U$, el salto térmico entre la temperatura media del colector y la temperatura ambiente $\left(T_{m}-T_{a}\right)$ y la irradiancia normal a la superficie del colector, $I_{n}$, según:

$$
\eta=\eta_{0}-U\left(T_{m}-T_{a}\right) /{ }^{I n}
$$

Utilizar colectores de tubos de vacío es clave para aprovechar el buen recurso invernal local, dado que su coeficiente de pérdidas de calor $(U)$ es casi cinco veces menor que los planos (U l y $5 \mathrm{~W} /$ $\mathrm{m}^{2} /{ }^{\circ} \mathrm{C}$ respectivamente), y, por lo tanto, es la mejor elección en climas fríos y operando con altas temperaturas $\left(90^{\circ} \mathrm{C}\right)$.

La figura 2 compara la curva de eficiencia $(\eta)$ típica de un colector plano con dos curvas de un mismo colector de tubos de vacío (Stickney $\&$ Soifer, 2006). Esta duplicidad se genera por considerar el área total bruta (gross, incluyendo espaciado entre tubos) o la de apertura (área proyectada del tubo exterior, $58 \mathrm{~mm} \times 1.800 \mathrm{~mm}$ ). Si bien el cálculo energético será equivalente en ambos casos, no ocurre lo mismo con la eficiencia, y entonces la comparación del colector plano y de tubos de vacío (tomando el área bruta), como se suele considerar en la literatura, conduce a resultados equivocados. De esta comparación se suele inferir la conveniencia de los colectores planos para usos hogareños, soslayando varios factores.

Primeramente, nótese de la ecuación de eficiencia (Ec.1) que la irradiancia normal al colector $\left(I_{n}\right)$ se encuentra dividiendo en el término de pérdidas. Siendo $U$ mayor en los colectores planos, se suelen tomar valores altos de $I_{n}$ (por ejemplo, $800 \mathrm{~W} /$ $\mathrm{m}^{2}$ ) con lo cual resultan reducidas las pérdidas. El mayor error conceptual aquí es comparar las curvas con el mismo $I_{n}$, siendo que el colector plano recibiría esta alta irradiación solo al mediodía, y en otros horarios, reducida por el coseno del ángulo azimutal. En cambio, el colector cilíndrico presenta un área normal constante (Juanicó, Di Lalla \& González, 2017, p. 190). Además, el colector de tubo de vacío tiene doble tubo, uno interno absorbedor y uno externo que garantiza el vacío. Las reflexiones de la radiación incidente sobre el tubo exterior generan un aumento adicional del área efectiva de captación, junto con la mayor captación de la radiación difusa sobre toda su circunferencia. Este efecto es relativamente mayor en días nublados, como es reconocido en la literatura. Estos dos últimos factores no serán computados en este estudio, para el cual además asumiremos en forma conservativa una eficiencia promedio constante a lo largo del año. Dos factores justifican esta hipótesis: 


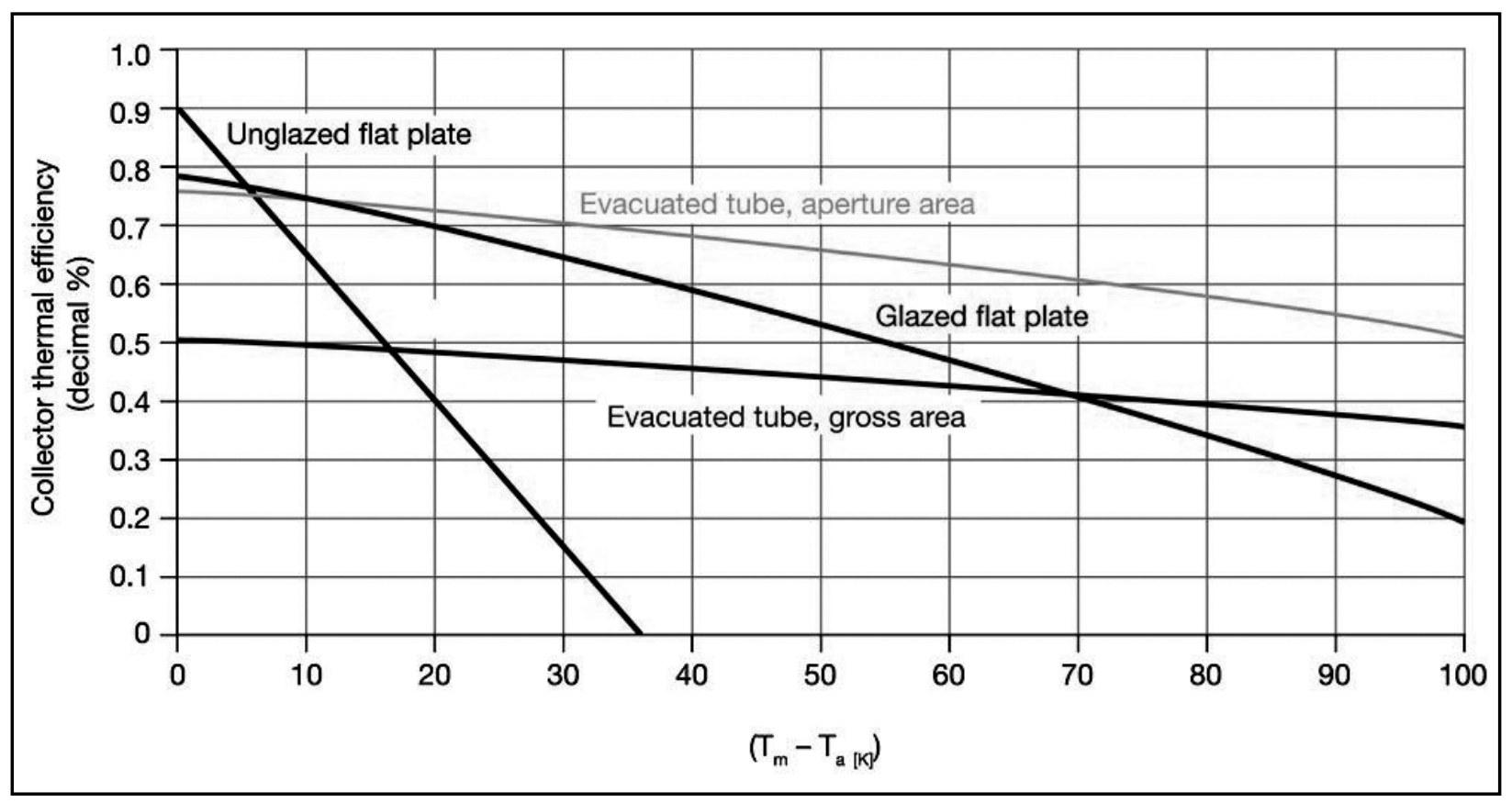

Figura 2. Eficiencia de colectores de tubos y planos, $\mathrm{I}_{\mathrm{n}}=800 \mathrm{~W} / \mathrm{m}^{2}$. Fuente: B. Stickney, \& B. Soifer, 2006

1.-En verano la temperatura ambiente diurna es alta $\left(T_{a}=25^{\circ} \mathrm{C}\right)$ y la de colectores también, $\mathrm{Tm}=90^{\circ} \mathrm{C}$, dando un salto térmico $T_{m}-T_{a}=65$ ${ }^{\circ} \mathrm{C}$; mientras que en invierno es menor $(\Delta \mathrm{T}=45$ ${ }^{\circ} \mathrm{C}$, con $T_{a}=5^{\circ} \mathrm{C}$ y $T_{\mathrm{m}}=50^{\circ} \mathrm{C}$ ) y así, las pérdidas de calor (véase Ec. 1) son menores, lo cual tiende a compensar la menor irradiación, In. Nótese que $T_{m}$ en invierno es significativamente

160 revista invi 33(93) : 153-172, agosto 2018 menor que $90^{\circ} \mathrm{C}$, dado que durante esta estación el reservorio pierde la energía almacenada. Esto se discutirá más adelante en tablas 4 y 5 para los dos tamaños de reservorios elegidos.

2.-El bajo coeficiente de pérdidas $(U)$ en colectores de vacío reduce la variación de la eficiencia ante cambios, tanto de $\Delta \mathrm{T}$ como de In, como muestra la figura 2. 
Por lo anterior, y según la curva de eficiencia del área de apertura $(0,1044 \mathrm{~m} 2$ por tubo) de la Figura 2, consideraremos una eficiencia promedio del $60 \%$, la cual equivale a una eficiencia del $40 \%$ basada en el área bruta. Esta eficiencia corresponde a un $\Delta \mathrm{T}=70^{\circ} \mathrm{C}$, que, siendo conservativos, abarca las condiciones de invierno y verano.

\section{MODELO TÉRMICO GENERAL}

Modelaremos el reservorio sobre las siguientes hipótesis simplificativas:

- El tanque se reviste con una capa uniforme de aislante $\left(U=0.1 \mathrm{~W} / \mathrm{m}^{2} /{ }^{\circ} \mathrm{C}\right)$

- La temperatura exterior (bajo tierra) es constante e igual a la media $T_{\text {suelo }}=8.4^{\circ} \mathrm{C}$

- Todos los términos temporales (potencias térmicas y variaciones de temperatura) se consideran a través de sus promedios mensuales.

- La temperatura del tanque se considera uniforme (gradientes despreciables)

Sobre estas hipótesis se puede representar la evolución temporal de la temperatura del reservorio $\left(d T_{r} / d t\right)$ mediante un modelo de capacitancias concentradas, en el cual su variación de energía interna surge (a partir de la ecuación de conservación de energía) del balance entre la potencia solar captada $\left(Q_{\text {solar }}\right)$ y la potencia perdida, tanto por pérdidas hacia el entorno $\left(Q_{\text {perdida }}\right)$ como por aportes a la calefacción $\left(Q_{\text {cale }}\right)$, como:

$$
M_{r} c\left(d T_{r}(t) / d t\right)=Q_{\text {solar }}(t)-\mathcal{Q}_{\text {perdida }}(t)-\mathcal{Q}_{\text {calef }}(t)(2)
$$

donde $M_{r}$ es la masa del reservorio y $c$ la capacidad calorífica del agua. Considerando solo promedios mensuales para modelar la evolución anual, obtenemos un sistema de 12 ecuaciones (similares a Ec.2) con 12 incógnitas: $T_{1}, T_{2} \ldots . T_{12}$, según:

$$
\begin{aligned}
& M_{r} c\left(d T_{1} / d t\right) \cong M_{r} c\left(T_{2}^{1}-T_{1}^{0}\right) / \Delta t=Q_{\text {solar }}{ }^{1}-Q_{\text {perdida }}{ }^{1}-Q_{\text {calef }}{ }^{1} \\
& M_{r} c\left(d T_{2} / d t\right) \cong \operatorname{Mrc}\left(T_{3}^{1}-T_{2}{ }^{1}\right) / \Delta t=Q_{\text {solar }}{ }^{2}-Q_{\text {perdida }}{ }^{2}-Q_{\text {calef }}{ }^{2} \\
& M_{r} c\left(d T_{12} / d t\right) \cong \operatorname{Mrc}\left(T_{1}^{1}-T_{12}{ }^{1}\right) / \Delta t=Q_{\text {solar }}{ }^{12}-Q_{\text {perdida }}{ }^{12}-Q_{\text {calef }}{ }^{12}(3-12)
\end{aligned}
$$

en donde se ha aproximado cada tasa temporal $(d T / d t)$ por su cociente incremental en el paso de tiempo (mensual) definido, $\left(T_{n+1}-T_{n}\right) / \Delta t$, transformando de este modo este sistema de ecuaciones diferenciales en otro algebraico. Además, al haber utilizado este esquema numérico explícito de un paso temporal (llamado aproximación de Euler), este sistema se puede resolver en forma sencilla por un método iterativo, ya que se despeja la temperatura del mes siguiente $\left(T_{n+1}\right)$ en cada ecuación del n-simo mes. Así, se comienza fijando la condición inicial del primer mes $\left(T_{1}^{0}\right)$ y se despeja en Ec. 3-1 el primer valor estimado para la temperatura del segundo mes $\left(T_{2}{ }^{1}\right)$, y así sucesivamente hasta llegar a la última (Ec. 3-12), en la cual se despeja el nuevo valor del primer mes $\left(T_{1}{ }^{1}\right)$; con este nuevo valor 
para el primer mes se repite el ciclo anterior hasta que el sistema converja a la solución, que describe el estacionario periódico anual del sistema.

Para resolver el sistema de ecuaciones (3), resta explicitar los tres términos de potencia $\left(Q_{\text {solar }}\right.$, $\left.\mathcal{Q}_{\text {perdida }} y \mathcal{Q}_{\text {cale }}\right)$ en cada mes. La potencia ganada $\left(\mathcal{Q}_{\text {solar }}\right)$ depende del sistema de colectores solares propuesto y del recurso solar de la locación escogida. Para un conjunto de $\mathrm{N}$ colectores de área de captación $A_{c}$ y eficiencia térmica instantánea $\eta(\mathrm{t})$, la potencia instantánea captada desde un flujo de irradiación normal al colector, $I_{n}(t)\left(\mathrm{W} / \mathrm{m}^{2}\right)$, viene dada por:

$$
Q_{\text {solar }}(t)=N A_{c} \eta(t) I_{n}(t)
$$

A los fines propuestos (cálculo de un reservorio), es razonable considerar aquí solo los promedios mensuales; según el mapa solar argentino ${ }^{9}$ determinamos el promedio mensual del recurso solar diario sobre el plano horizontal en cada n-simo mes, $G_{n}$ (kWh/m²/día) (Figura la) y fijamos la eficiencia promedio $\eta=60 \%$ (del área de apertura, representa un salto térmico de $70^{\circ} \mathrm{C}$ según Figura 2, y una eficiencia del 40\% según el área bruta). Integrando en cada mes la ecuación anterior, calculamos el promedio mensual de la energía captada en el n-simo mes, $E_{\text {solar }}$ :

$$
\mathrm{E}_{\text {solar }}{ }^{\mathrm{n}}=\mathrm{N} A_{\mathrm{c}} \eta \alpha_{\mathrm{n}} \mathrm{G}_{\mathrm{n}}
$$

donde aparece un nuevo factor, $\alpha_{n}$, que depende de la latitud, día del año y del ángulo $\varphi$ de elevación del colector. El factor $\alpha_{n}$ expresa la relación entre el flujo de energía solar irradiada sobre el plano horizontal $\left(G_{n}\right)$ y sobre el plano del colector elevado un cierto ángulo $\varphi$. Este parámetro puede ser calculado a partir de la trayectoria solar aparente con programas disponibles en la literatura. Para un colector de tubos cilíndricos orientados al norte en Bariloche $\left(42^{\circ} \mathrm{S}\right)$, la tabla 2 resume los valores de $\alpha_{n} \times G_{n}$ calculados para cinco ángulos $\varphi$; se observa que la energía anual colectada se maximiza para $\varphi=52^{\circ}$ (latitud $+10^{\circ}$ ). Sin embargo, cuando se plantea su uso para satisfacer la demanda de calefacción (máxima en invierno), sugiere considerar ángulos de mayor captación invernal: por ejemplo, con $\varphi=70^{\circ}$ se obtiene $7 \%$ más en invierno.

Nótese la marcada diferencia entre la irradiación anual recibida sobre un plano horizontal $(G)$ y sobre planos inclinados de $45^{\circ}$ a $70^{\circ}$ (cercano al doble). Este efecto se potencia en invierno, donde la radiación en un plano a $70^{\circ}$ es más del triple.

Considerando las potencias perdidas al entorno en cada mes $\left(Q_{\text {perdida }}^{n}\right)$, el material aislante (de espesor e y conductividad térmica $k$ ) brindará una cierta aislación, caracterizada por su coeficiente de transmisión de calor $U\left(\mathrm{~W} / \mathrm{m}^{2 \circ} \mathrm{C}\right)$, según:

$$
U=k / e
$$


TABLA 2. PROMEDIOS MENSUALES DE $\alpha_{\eta} X G_{\eta}\left(K W H / M^{2} /\right.$ DÍA) PARA DIFERENTES $\varphi$.

\begin{tabular}{|lrrrrrr|}
\hline & $0^{\circ}$ & $30^{\circ}$ & $42^{\circ}$ & $52^{\circ}$ & $60^{\circ}$ & $7^{\circ}$ \\
\hline Enero & 7,50 & 11,09 & 11,73 & 11,87 & 11,72 & 11,22 \\
\hline Febrero & 6,50 & 10,23 & 10,98 & 11,24 & 11,20 & 10,85 \\
\hline Marzo & 4,50 & 7,66 & 8,38 & 8,71 & 8,77 & 8,62 \\
\hline Abril & 3,00 & 5,96 & 6,73 & 7,14 & 7,32 & 7,34 \\
\hline Mayo & 2,00 & 4,70 & 5,46 & 5,92 & 6,15 & 6,27 \\
\hline Junio & 1,40 & 3,65 & 4,30 & 4,71 & 4,92 & 5,06 \\
\hline Julio & 1,50 & 3,79 & 4,45 & 4,85 & 5,06 & 5,19 \\
\hline Agosto & 2,50 & 5,40 & 6,19 & 6,64 & 6,86 & 6,94 \\
\hline Septiembre & 4,00 & 7,27 & 8,07 & 8,47 & 8,60 & 8,53 \\
\hline Octubre & 5,50 & 8,81 & 9,50 & 9,77 & 9,76 & 9,49 \\
\hline Noviembre & 7,00 & 10,63 & 11,31 & 11,50 & 11,40 & 10,96 \\
\hline Diciembre & 7,50 & 11,09 & 11,73 & 11,87 & 11,72 & 11,22 \\
\hline Anual & 1.605 & 2.740 & 3.000 & 3.118 & 3.142 & 3.088
\end{tabular}

Fuente:Autores.

De aquí, podemos calcular la potencia perdida en el n-simo mes como (Bergman, Lavine, Incropera, \& DeWitt, 2011):

$$
Q_{\text {perdida }}^{n}=U A_{r}\left(T_{n}-T_{\text {suelo }}\right)
$$

donde $A_{r}$ es el área total del reservorio. Para calcular la energía perdida en el n-simo mes, integramos la ecuación anterior considerando la temperatura del reservorio $\left(T_{n}\right)$ (y tomando $\Delta t_{n}=28,30$ o 31 días) como:

$$
E_{\text {perdida }}^{n}=U A_{r}\left(T_{n}-T_{\text {suelo }}\right) \Delta t_{n}
$$

Similarmente, integrando la potencia de calefacción requerida a lo largo de cada mes, obtenemos la energía extraída para calefacción en el $n$-simo mes:

$$
E_{\text {calef }}^{n}=Q_{\text {calef }}^{n} \Delta t^{n}
$$

donde la potencia de calefacción en cada mes es un dato (Tabla 1), calculado a partir de los promedios

revista invi 33(93) : 153-172, agosto 2018163 
de temperatura mensuales (ver Figura 1b). Sustituyendo las ecuaciones anteriores (5-9) en el sistema anterior (3-n), obtenemos un nuevo sistema que se resuelve en secuencia, despejando $\left(T_{1}\right.$, $\left.T_{2}, \ldots T_{12}\right)$ de:

$$
\begin{aligned}
& M_{r} c\left(T_{2}^{1}-T_{1}{ }^{0}\right)=E_{\text {solar }}{ }^{1}-E_{\text {perdida }}{ }^{1}-E_{\text {calef }} \\
& M_{r} c\left(T_{3}^{1}-T_{2}^{1}\right)=E_{\text {solar }}{ }^{2}-E_{\text {perdida }}{ }^{2}-E_{\text {calef }}{ }^{2} \\
& M_{r} c\left(T_{1}^{1}-T_{12}{ }^{1}\right)=E_{\text {solar }}{ }^{12}-E_{\text {perdida }}{ }^{12}-E_{\text {calef }}{ }^{12}
\end{aligned}
$$

\section{Resultados y discusión}

Consideramos un buen nivel de aislación $(U=0,1$ $\mathrm{W} / \mathrm{m}^{2 \circ} \mathrm{C}$ ) para el reservorio, resoluble con $35 \mathrm{~cm}$ de EPS $(k=0.035 \mathrm{~W} / \mathrm{m} \cdot \mathrm{K}$ ) (Laboratorio de Ambiente Humano y Vivienda, 2017) y colectores heat pipe (área apertura $2.088 \mathrm{~m}^{2}$ y su eficiencia 60\%) instalados un ángulo $\varphi=52^{\circ}$. Los dos parámetros de diseño del sistema: masa del reservorio y número de colectores, se calculan para diferentes pares soluciones, siendo posible utilizar menos colectores con un reservorio más grande y viceversa, a condición de que la temperatura del reservorio se mantenga dentro de los límites $33^{\circ} \mathrm{C}$ a $85^{\circ} \mathrm{C}$.

La tabla 3 resumen los resultados obtenidos, donde $D_{r}$ y $H_{r}$ son el diámetro y la altura del tanque. Se considera el diámetro y la altura iguales para minimizar el área. Se observa que no es posible satisfacer toda la demanda con cuatro colectores, debido a que la energía solar captada a lo largo del año no alcanza a cubrir la demanda de calefacción (13.000 kWh). Nótese que esta condición es independiente del tamaño de reservorio elegido. Aumentando mínimamente el área de colectores (5 colectores) es posible satisfacer el 100\% de la demanda; sin embargo el tamaño del tanque resultante es de $63 \mathrm{~m}^{3}$ con un área de pérdida de calor al suelo de $87 \mathrm{~m}^{2}$, resultando en una eficiencia de $79 \%$, lo cual significa que pierde hacia el entorno el 21\% del calor recibido debido a su gran tamaño. Se muestra también en la tabla 3 el número de meses en los cuales es necesario limitar la temperatura del reservorio a su valor máximo $\left(85^{\circ} \mathrm{C}\right)$ debido al exceso de energía captada.

En la tabla 3 se muestra el análisis de sensibilidad variando el número de colectores, con el cual varía fuertemente el tamaño del tanque. Aumentando el número de colectores de cinco a ocho, se reduce el volumen del tanque 6 veces y su área de pérdidas cuatro veces. Un colector más permitiría eliminar el tanque, pero entonces el sistema se volvería cautivo de las condiciones meteorológicas; el error surge de olvidar que consideramos solo promedios mensuales, válidos solo con un reservorio.

Nótese que el tanque más pequeño presenta un rendimiento del $92 \%$ contra $79 \%$ del mayor debido a su menor área de perdida de calor, obtenida a partir de un número de colectores que prácticamente 
TABLA 3. CONJUNTO DE PARES SOLUCIONES $\left(\varphi=52^{\circ}\right)$.

\begin{tabular}{|c|c|c|c|c|c|}
\hline $\begin{array}{r}\text { Colectores } \\
\text { (\#) }\end{array}$ & $\begin{array}{r}A_{r} \\
\left(\mathrm{~m}^{2}\right)\end{array}$ & $\begin{array}{r}D_{r}=H_{r} \\
(\mathrm{~m})\end{array}$ & $\begin{array}{r}V_{r} \\
\left(\mathrm{~m}^{3}\right)\end{array}$ & $\begin{array}{l}\text { Exceso E. } \\
\text { (\# meses) }\end{array}$ & $\eta_{\text {tanque }}$ \\
\hline 4 & -- & -- & -- & --- & --- \\
\hline $5-A$ & 87 & 4,3 & 63 & 3 & $79 \%$ \\
\hline 6 & 65 & 3,7 & 40 & 6 & $83 \%$ \\
\hline 7 & 40 & 2,9 & 19 & 7 & $89 \%$ \\
\hline $8-B$ & 27 & 2,4 & 11 & 9 & $92 \%$ \\
\hline 9 & 0 & 0 & 0 & 12 & -- \\
\hline
\end{tabular}

Fuente:Autores.

solventan esta demanda mes a mes, sin requerir casi almacenamiento. Este concepto se muestra en detalle en el análisis del balance térmico anual del sistema, según las dos configuraciones extremas; A) 5 colectores y tanque $63 \mathrm{~m}^{3}$; B) 8 colectores y tanque $11 \mathrm{~m}^{3}$.

La tabla 4 resume el caso A. Para cada mes del año, se indican los términos de energía solar captada, entregada al sistema de calefacción, perdidas de calor al entorno y el balance de los anteriores, junto con la temperatura del reservorio. Nótese que siendo este balance positivo, no implica necesariamente que esta energía pueda ser acumulada en el reservorio, toda vez que existe una temperatura máxima de trabajo $\left(85^{\circ} \mathrm{C}\right)$. Desde octubre hasta marzo el reservorio acumula energía, mientras que de abril a setiembre el balance es negativo, y en consecuencia la mayor demanda de calefacción se satisface a expensas del reservorio. Durante los meses de enero a marzo el reservorio no es capaz de almacenar la energía neta generada, por lo cual su temperatura se debe limitar a $85^{\circ} \mathrm{C}$, pudiendo este excedente aprovecharse por ejemplo, para calefaccionar una piscina. En la última fila de la tabla 4 se muestran los balances anuales y el rendimiento del reservorio.

La tabla 5 resume el caso B. A diferencia del caso anterior, con la excepción de los meses de junio y julio, la ganancia solar es mayor a las extracciones de calor durante diez meses, durante los cuales el reservorio se mantiene a $85^{\circ} \mathrm{C}$, desechando el exceso de calor. Esto genera un interesante excedente, útil por ejemplo para satisfacer la demanda doméstica de agua caliente. La alta eficiencia obtenida en el sistema (92\%) sugiere que no es necesario mayor aislamiento térmico en el tanque, como 
TABLA 4. EVOLUCIÓN ANUAL DEL SISTEMA (A).

\begin{tabular}{|lrrrrr|}
\hline & $\begin{array}{c}\mathrm{E}_{\text {solar }} \\
(\mathrm{MJ})\end{array}$ & $\begin{array}{r}\mathrm{E}_{\text {calef }} \\
(\mathrm{MJ})\end{array}$ & $\begin{array}{r}\mathrm{E}_{\text {pérdida }} \\
(\mathrm{MJ})\end{array}$ & $\begin{array}{r}\mathrm{E}_{\text {balance }} \\
(\mathrm{MJ})\end{array}$ & $\begin{array}{r}\mathrm{T} \\
\left({ }^{\circ} \mathrm{C}\right)\end{array}$ \\
\hline Enero & 8.298 & 1.568 & 1.510 & 5.220 & 85,0 \\
\hline Febrero & 7.097 & 1.374 & 1.615 & 4.109 & 85,0 \\
\hline Marzo & 6.089 & 2.661 & 1.788 & 1.640 & 85,0 \\
\hline Abril & 4.830 & 3.679 & 1.730 & -579 & 82,8 \\
\hline Mayo & 4.138 & 5.132 & 1.736 & -2.730 & 72,4 \\
\hline Junio & 3.186 & 5.565 & 1.445 & -3.823 & 57,8 \\
\hline Julio & 3.390 & 6.225 & 1.153 & -3.988 & 42,6 \\
\hline Agosto & 4.642 & 5.988 & 798 & -2.144 & 34,4 \\
\hline Septiembre & 5.730 & 4.921 & 588 & 221 & 35,3 \\
\hline Octubre & 6.830 & 4.134 & 1.510 & 1.185 & 39,8 \\
\hline Noviembre & 7.780 & 3.081 & 709 & 3.990 & 55,0 \\
\hline Diciembre & 8.298 & 2.471 & 1.088 & 4.739 & 73,1 \\
\hline ANUAL & 47.401 & 46.801 & 12.362 & & \\
\hline ANUAL & & $79 \%$ & $21 \%$ & & \\
\hline
\end{tabular}

Fuente:Autores.

fue propuesto para el proyecto de Galway con 60 $\mathrm{cm}$ de EPS (Colclough \& Griffiths, 2016).

Nótese que la capacidad intrínseca de almacenamiento en ambos reservorios es limitada. El tanque mayor $\left(63 \mathrm{~m}^{3}\right)$ podría almacenar un máximo de energía útil de $3.822 \mathrm{kWh}$, los cuales solventarían la demanda de calefacción más exigente $(55 \mathrm{kWh}$ diarios en julio) durante 70 días, y durante solo 12 días para el tanque menor (11 $\left.\mathrm{m}^{3}, 667 \mathrm{kWh}\right)$.
Sin embargo, debido a que los colectores solares realizan significativos aportes de energía durante este periodo, el sistema térmico puede satisfacer la demanda de calefacción durante todo el intervalo de balance negativo (5 meses en caso A y 2 meses en caso B), como muestran las tablas 4 y 5 . Es por esto importante considerar al reservorio de calor acoplado al sistema solar, pudiendo este potenciar apreciablemente a aquel. 
TABLA 5. BALANCE TÉRMICO ANUAL DEL SISTEMA (B).

\begin{tabular}{|c|c|c|c|c|c|}
\hline & $\begin{array}{l}E_{\text {solar }} \\
(\mathrm{MJ})\end{array}$ & $\begin{array}{l}E_{\text {calef }} \\
(\mathrm{MJ})\end{array}$ & $\begin{array}{c}\mathrm{E}_{\text {pérdida }} \\
(\mathrm{MJ})\end{array}$ & $\begin{array}{l}E_{\text {balance }} \\
(\mathrm{MJ})\end{array}$ & $\begin{array}{r}\mathrm{T} \\
\left({ }^{\circ} \mathrm{C}\right)\end{array}$ \\
\hline Enero & 13.277 & 1.568 & 557 & 11.151 & 85,0 \\
\hline Febrero & 11.355 & 1.374 & 503 & 9.479 & 85,0 \\
\hline Marzo & 9.742 & 2.661 & 557 & 6.524 & 85,0 \\
\hline Abril & 7.728 & 3.679 & 539 & 3.510 & 85,0 \\
\hline Mayo & 6.622 & 5.132 & 557 & 932 & 85,0 \\
\hline Junio & 5.098 & 5.565 & 539 & -1.006 & 62,9 \\
\hline Julio & 5.425 & 6.225 & 397 & -1.197 & 36,7 \\
\hline Agosto & 7.427 & 5.988 & 206 & 1.233 & 63,7 \\
\hline Septiembre & 9.168 & 4.921 & 389 & 3.858 & 85,0 \\
\hline Octubre & 10.928 & 4.134 & 557 & 6.236 & 85,0 \\
\hline Noviembre & 12.448 & 3.081 & 539 & 8.828 & 85,0 \\
\hline Diciembre & 13.277 & 2.471 & 557 & 10.249 & 85,0 \\
\hline ANUAL & 75.842 & 46.801 & 4.243 & & \\
\hline ANUAL & & $92 \%$ & $8 \%$ & & \\
\hline
\end{tabular}

Fuente:Autores.

Cabe destacar que en todos los casos tratados aquí se establece la condición de satisfacer el 100\% de la demanda de calefacción. El modelo aquí propuesto puede aplicarse a otras localizaciones y sistemas constructivos, requiere de conocer la demanda de calefacción, las temperaturas medias mensuales, la radiación solar promedio mensual sobre el plano horizontal, y los coeficientes angulares para la radiación sobre el plano del colector. Estos últimos se obtienen de programas estándar de trayectorias solares, con especificaciones para la localización propuesta. 


\section{Análisis de costos}

Analizaremos los costos en ambas soluciones límites. La tabla 6 resume los costos desagregados por ítem en ambos casos. Los ítems que varían el costo entre la opción A y B son los colectores, el tanque y su aislamiento térmico, y otros gastos para la instalación. Se observa que el incremento en la capacidad solar instalada (caso B vs. A) produce un significativo ahorro en el tanque y su aislación. Los ítems que no dependen del tamaño de la demanda definida representan el $47 \%$ del costo total en la opción más económica (B). En el caso de demandas mayores, el caso B presenta mayor potencial de ahorro por economía de escala, respecto al caso A.

La optimización económica nos ha llevado hacia reservorios pequeños; sin embargo, estos sistemas están más al límite de cubrir la demanda en la estación invernal, por lo cual recalculamos el caso B con optimización del ángulo $(\varphi)$ de captación solar. Instalando los colectores $\varphi=70^{\circ}$ se obtiene un mejor desempeño, siendo la temperatura mínima del reservorio (en julio) de $61,4^{\circ} \mathrm{C}$ (antes $36,7^{\circ} \mathrm{C}$ $\operatorname{con} \varphi=52^{\circ}$ ).
TABLA 6. COSTOS DESAGREGADOS PARA SOLUCIONES LÍMITES (A Y B).

\begin{tabular}{lll} 
& A & B \\
& (USD) & (USD) \\
\hline Colectores & 2.500 & 4.000 \\
\hline Instalación colectores & 1.000 & 1.000 \\
\hline Tanque & 14.700 & 1.400 \\
\hline Aislante (50 USD $\left./ \mathrm{m}^{2}\right)$ & 4.350 & 1.350 \\
\hline Intercambiadores (2) & 400 & 400 \\
\hline Bomba (2) & 1.000 & 1.000 \\
\hline Controlador & 200 & 200 \\
Cañerías & 300 & 300 \\
\hline Otros & 2.500 & 2.000 \\
\hline Total & $\mathbf{2 7 . 0 0 0}$ & $\mathbf{1 1 . 6 5 0}$ \\
\hline
\end{tabular}

Fuente:Autores. 


\section{Conclusiones}

Se investigó la factibilidad técnica-económica de la calefacción con energía solar de viviendas en Bariloche, en base a colectores heat pipe y acumulación térmica en un gran reservorio de agua. Se adaptaron conceptos utilizados en países desarrollados. En comparación con éstos, para temperatura media anual similar, los resultados demuestran que es posible lograr notables reducciones de tamaño y costo de las instalaciones, debido a cuatro factores:

- Climático, con inviernos más templados y veranos más fríos

- Recurso solar más distribuido y con aportes invernales importantes

- Colectores heat pipe, que maximizan la captación del recurso solar

- Tanques pequeños, enterrados y bien aislados, obtienen mucho menor coste y eficiencia que una configuración de gran tanque.

La herramienta de modelado desarrollada es sencilla de programar en hoja de cálculo ya que se reduce a un conjunto de ecuaciones algebraicas con un solo término de iteración (mes a mes). Estas son resolubles manualmente en pocas iteraciones, o también, por ejemplo, se puede programar con una subrutina "macro" en Excel. Esta herramienta permite optimizar todos los parámetros térmico-solares del sistema: i) volumen del tanque; ii) aislación del tanque; iii) número de colectores y iv) su ángulo de elevación. Todos ellos actúan fuertemente relacionados y solo se pueden ponderar calculando la solución global (estacionario periódico del sistema). El estudio numérico, junto con el análisis económico, arrojó resultados muy diferentes de los históricos diseños alemanes y canadienses, centrados en enormes reservorios estacionales. Estos proyectos pueden entonces ser discutidos en sus distintos componentes optimizables mediante este nuevo modelo térmico integral. Por ejemplo: ies conveniente un enorme tanque pero que resigna aislación?; ¿cuál es el espesor óptimo de aislación?; ¿qué tipo de colectores instalar?; y ¿cuál su ángulo de elevación óptimo?

Para el caso estudiado se obtuvo una condición de mínimo número de colectores solares (5 de 20 tubos heat pipe), resultando un tanque acumulador de $63 \mathrm{~m}^{3}$. A través de un análisis de sensibilidad se obtuvieron distintas configuraciones, y se observó que sumando 3 colectores, se puede reducir 6 veces el volumen del reservorio y 56\% la inversión total. Este resultado es interesante, siendo que se contrapone al diseño tradicional de un enorme reservorio estacional, y se basa en los cuatro factores antes explicados. También, se observa que incrementando el ángulo de elevación de los colectores por encima del tradicionalmente empleado (latitud $+10^{\circ}$ ), se obtiene un mejor desempeño global. Todo esto demuestra la potencialidad de esta herramienta de cálculo para optimizar un caso dado. 
En el caso optimizado con mayor número de colectores y menor tanque se obtuvo un exceso de energía durante 10 meses al año, que permitiría satisfacer parcialmente la demanda de agua caliente sanitaria (ACS). En este trabajo simulamos una demanda de $13.000 \mathrm{kWh}$, representativo de una vivienda de $100 \mathrm{~m}^{2}$ con aislamiento térmico moderado pero que también representa una vivienda económica de $50 \mathrm{~m}^{2}\left(230 \mathrm{kWh} / \mathrm{m}^{2}\right)$ u otra con diseño bioclimático de $200 \mathrm{~m}^{2}\left(65 \mathrm{kWh} / \mathrm{m}^{2}\right)$. Es posible además extender el análisis a otros casos que representen una demanda similar aunque solo para ACS: los sistemas propuestos aquí cubrirían la demanda anual de ACS de 21 personas (50 litros diarios y $\Delta \mathrm{T}=30^{\circ} \mathrm{C}$ ).

Queremos remarcar que este modelo es válido para diferentes patrones climáticos (caracterizados por su demanda de calefacción y curva de distribución), diferentes recursos solares (caracterizados por su curva de distribución), diferentes colectores solares (caracterizados por su eficiencia promedio y área de captación) y su ángulo de elevación (caracterizado por factores $\alpha_{n}$ ).

Esta tecnología podría generalizarse para la región del Cono Sur para satisfacer la demanda de calefacción, donde se emplean habitualmente fuentes no renovables y/o con alto impacto medioambiental y/o altamente contaminantes como ser leña y gas en Chile y Argentina, y generadoras termoeléctricas en Uruguay. En momentos en que se observa una decidida difusión de los colectores solares en la región, pero casi limitada a la provisión de ACS, esta propuesta podría abrir nuevos mercados solares.

\section{Agradecimientos}

El presente trabajo ha sido financiado por el proyecto CONICET PIP 11220130100048CO "Uso eficiente de energía y aprovechamiento del recurso solar en la Patagonia Andina"; y por el proyecto de la Universidad Nacional del Comahue PIN I B191 "Medioambiente y sociedad: peligros naturales y vulnerabilidad en poblaciones de Patagonia Andina".

\section{Referencias bibliográficas}

Bär, K., Rühaak, W., Welsch, B., Schulte, D., Homuth, S., \& Sass. I. (2015). Seasonal high temperature heat storage with medium deep borehole heat exchangers. Energy Procedia, 76, 351-360. https:// doi.org/10.1016/j.egypro.2015.07.841.

Bauer, D., Marx, R., Nußbicker-Lux, J.; Ochs, F., Heidemann, W. \& Müller-Steinhagen, H. (2010). German central solar heating plants with seasonal heat storage. Solar Energy, 84(4), 612-623. https:// doi.org/10.1016/j.solener.2009.05.013 
Bergman, T.L., Lavine, A.S., Incropera, F.P., \& DeWitt, D.P. (2011). Fundamentals of heat and mass transfer (7th ed.). Hoboken, NJ: Wiley.

BOWA Solution. (2017). Recuperado de https://bowasolution.es.aliexpress.com/store/1333457

Colclough, S. \& Griffiths, P. (2016). Financial analysis of an installed small scale seasonal thermal energy store. Renewable Energy, 86, 422-428. https://doi. org/10.1016/j.renene.2015.08.032.

Díaz, C. y Czajkowski, J. (2006). Auditorías energéticas en viviendas de interés social en Río Grande, Tierra del fuego. Avances en Energías Renovables y Medio Ambiente, 10, 07.33-07.38.

González, A.D. (2009). Energy subsidies in Argentina lead to inequalities and low thermal efficiency. Energies, 2(3), 769-788. https://doi.org/10.3390/ en20300769.

(2013). Management of disaster risks derived from very large fuel subsidies to natural gas in Argentina. En Climate change and disaster risk management. Climate change management, (pp. 463-473). Berlin, Heidelberg: Springer. https:// doi.org/10.1007/978-3-642-31110-9_30.

(2014). Casas confortables con mínimo uso de energía: estudio de casos prácticos para Argentina y Chile. San Carlos de Bariloche: Autor. Recuperado de http://www.ipatec.conicet.gob.ar/casas-confortables-con-minimo-uso-de-energia-estudiode-casos-practicos-para-argentina-y-chile/.
González, A.D., Carlsson-Kanyama, A., Crivelli, E.S., \& Gortari, S. (2007) Residential energy use in onefamily households with natural gas provision in a city of the Patagonian Andean region. Energy Policy, 35(4), 2141-2150. https://doi.org/10.1016/j. enpol.2006.07.004.

Grossi Gallegos, H. y Righini, R. (2007). Atlas de energía solar de la República Argentina. Argentina: Secretaría de Ciencia y Tecnología y Universidad Nacional de Luján.

Juanicó, L. \& González, A.D. (2008a). Thermal efficiency of natural gas balanced-flue space heaters: measurements for commercial devices. Energy and Buildings, 40(6), 1067-1073. https://doi. org/10.1016/j.enbuild.2007.10.007

(2008b). Savings in natural gas consumption by doubling thermal efficiencies of balanced-flue space heaters. Energy and Buildings, 40(8), 1479-1486. https://doi.org/10.1016/j. enbuild.2008.02.002.

Juanicó, L.E., Di Lalla, N., \& González, A.D. (2017). Full thermal-hydraulic and solar modeling to study low-cost solar collectors based on a single long LDPE hose. Renewable and Sustainable Energy Reviews, 73, 187-195. https://doi.org/10.1016/j. rser.2017.01.126.

Laboratorio de Ambiente Humano y Vivienda. (2017). Conductividad térmica de materiales. Recuperado de http://www.mendoza-conicet.gob.ar/lahv/soft/ resistencia/ 
Rad, F. \& Fung, A. (2016). Solar community heating and cooling system with borehole thermal energy storage - Review of systems. Renewable Sustainable Energy Reviews, 60, 1550-1561. https://doi. org/10.1016/j.rser.2016.03.025.

Servicio Meteorológico Nacional. (2017). Caracterización: Estadísticas de largo plazo. Recuperado de https://www.smn.gob.ar/caracterizaci\%C3\%B3nestad\%C3\%ADsticas-de-largo-plazo.

Sibbitt, B., Mcclenahan, D., Djebbar, R., Thornton, J., Wong, B., Carriere, J.\& Kokko, J. (2012). The performance of a high solar fraction seasonal storage district heating system - five years of operation. Energy Procedia 30, 856-865. https://doi. org/10.1016/j.egypro.2012.11.097.

Stickney, B. \& Soifer, B. (2006). Collector efficiency: second order curves [gráfico]. Recuperado de http:// solarprofessional.com/articles/products-equipment/solar-heating/solar-thermal-hydronics/ page/0/4\#.WzFmlKfOk2z. 